\title{
Addressable Nanoantennas with Cleared Hotspots for Single- Molecule Detection on a Portable Smartphone Microscope
}

Kateryna Trofymchuk ${ }^{1,2_{\dagger} *}$, Viktorija Glembockyte ${ }^{1_{\dagger}} *$, Lennart Grabenhorst ${ }^{1}$, Florian Steiner ${ }^{1}$, Carolin Vietz $^{2}$, Cindy Close ${ }^{1}$, Martina Pfeiffer ${ }^{1}$, Lars Richter ${ }^{2}$, Max L. Schütte ${ }^{2}$, Florian Selbach ${ }^{1}$, Renukka Yaadav ${ }^{1}$, Jonas Zähringer ${ }^{1}$, Qingshan Wei ${ }^{3}$, Aydogan Ozcan ${ }^{4}$, Birka Lalkens ${ }^{5}$, Guillermo P. Acuna ${ }^{6}$, and Philip Tinnefeld ${ }^{1 *}$

\footnotetext{
${ }^{1}$ Department of Chemistry and Center for NanoScience, Ludwig-Maximilians-Universität München, Butenandtstr. 5-13, 81377 München, Germany

${ }^{2}$ Institute for Physical and Theoretical Chemistry - NanoBioScience and Braunschweig Integrated Centre of Systems Biology (BRICS), Technische Universität Braunschweig, Braunschweig, Germany

${ }^{3}$ Department of Chemical and Biomolecular Engineering, North Carolina State University, Raleigh, NC, 27695, United States

${ }^{4}$ Electrical \& Computer Engineering Department, Bioengineering Department, California NanoSystems Institute (CNSI), and Department of Surgery, University of California, Los Angeles, California 90095, United States

${ }^{5}$ Institut für Halbleitertechnik, Laboratory for Emerging Nanometrology LENA, TU Braunschweig, Langer Kamp 6a/b, 38106 Braunschweig, Germany

${ }^{6}$ Département de Physique - Photonic Nanosystems, Université de Fribourg - Faculté des Sciences et Médicine Chemin de Musée

3, CH-1700 Fribourg, Switzerland
}

$\dagger$ These authors contributed equally

The advent of highly sensitive photodetectors ${ }^{1,2}$ and the development of photostabilization strategies ${ }^{3}$ made detecting the fluorescence of a single molecule a routine task in many labs around the world. However, to this day, this process requires cost-intensive optical instruments due to the truly nanoscopic signal of a single emitter. Simplifying single-molecule detection would enable many exciting applications, e.g. in point-of-care diagnostic settings, where costly equipment would be prohibitive. ${ }^{4}$ Here, we introduce addressable NanoAntennas with Cleared HOtSpots (NACHOS) that are scaffolded by DNA origami nanostructures and can be specifically tailored for the incorporation of bioassays. Single emitters placed in the NACHOS emit up to 461-fold brighter enabling their detection with a customary smartphone camera and an 8-US-dollar objective lens. To prove the applicability of our system, we built a portable, battery-powered smartphone microscope and successfully carried out an exemplary single-molecule detection assay for DNA specific to antibiotic-resistant Klebsiella pneumonia „on the road “.

Early detection of disease biomarkers generally requires high sensitivity enabled by molecular amplification mechanisms ${ }^{5-9}$ or physical signal enhancement of commonly used fluorescence signals. ${ }^{10-13}$ Physical fluorescence signal enhancement could enable sensitivity improvement, detection of singlemolecules on cost-effective and mobile devices and therefore help to distinguish specific signals against an unavoidable background of impurities even in low-resource settings. Fluorescence from emitters such as fluorescent dyes can be enhanced using plasmonic nanoantennas, ${ }^{14-16}$ and the challenge of placing quantum emitters in their hotspots was overcome using DNA origami as constructing material. ${ }^{17,18}$ The immense requirements for small, defined and rigid gaps between the gold or silver nanoparticles forming the gap in the nanoantenna aggravated the usability of the space between the nanoparticles for a biosensing assay that could thus far only be realized with mono-particle antennas and low enhancement values. ${ }^{19}$

In this work, we introduce NACHOS that enable high fluorescence signal amplification and use them for a single-molecule diagnostic assay on a portable and inexpensive smartphone microscope. A novel threedimensional DNA origami structure was designed (Fig. 1a) and folded from an M13mp18-derived scaffold strand and complementary staple strands (Supplementary Tables 1-3). The NACHOS origami design uses two pillars to attach silver nanoparticles and creates the plasmonic hotspot at the bifurcation in the gap between the two pillars and the nanoparticles (see DNA origami sketches in Fig. 1a and full NACHOS structure in Fig. 
1b). Thus, the space of the hotspot, i.e. between the nanoparticles is left free for placing baits and for binding targets as needed for nucleic acid bioassays. For immobilization, the DNA origami structure is equipped with a rigid cross-like shaped base (approximately $35 \mathrm{~nm}$ by $33 \mathrm{~nm}$, Supplementary Figures 1 and 2) that contains six biotin-modified staples strands (Supplementary Table 3) used for immobilization on BSA-biotin coated coverslips via biotin-NeutrAvidin interactions (Fig. 1b). The two pillars of the DNA origami structure (total height $\sim 83 \mathrm{~nm}$ ) each contain six protruding staple strands $\left(\mathrm{A}_{20}\right.$, Supplementary Table 3$)$ which provide anchor points for binding DNA $\left(\mathrm{T}_{20}\right)$-functionalized $100 \mathrm{~nm}$ silver nanoparticles (Fig. 1b). The estimated distance between the nanoparticles is $\sim 12 \mathrm{~nm}$.

A transmission electron microscopy (TEM) image of an exemplary nanoantenna produced via solution synthesis is shown in Fig. 1c (see Materials and Methods section for details on magnetic bead-based solution synthesis). We evaluated the signal amplification that can be achieved in this DNA origami nanoantenna design by incorporating an Alexa Fluor 647-labelled DNA staple strand (Supplementary Table 3) directly into the plasmonic hotspot of the nanoantenna. Single-molecule fluorescence transients of the dye (Fig. 1d, Supplementary Figure 3) were recorded on a confocal microscope for the DNA origami sample without nanoparticles (orange) as well as for NACHOS containing two $100 \mathrm{~nm}$ silver nanoparticles attached to the DNA origami after immobilization on the coverslip (blue, see Materials and Methods section for NACHOS synthesis on the coverslip). Single-step photobleaching confirms that the detected signal originates from a single fluorescent molecule. The fluorescence enhancement obtained for each nanoantenna was calculated by comparing the intensity of Alexa Fluor 647 in the NACHOS to the mean intensity of Alexa Fluor 647 in the reference structure without nanoparticles. Fluorescence enhancement values of up to 417 -fold could be achieved in the new nanoantenna design (Fig. 1e). The broad fluorescence enhancement distribution reflects some heterogeneity with regard to nanoparticle size, shape and orientation, and also includes a subpopulation of monomer nanoantennas. Care was taken that all fluorescent molecules incorporated in the DNA origami nanoantennas were included in the analysis to obtain a representative distribution of fluorescence enhancement values in Fig. 1e. Most importantly, we note that increasing the accessibility of the hotspot region did not compromise the fluorescence enhancement values which are slightly higher than previously reported values for more compact nanoantenna designs. ${ }^{18,20,21}$
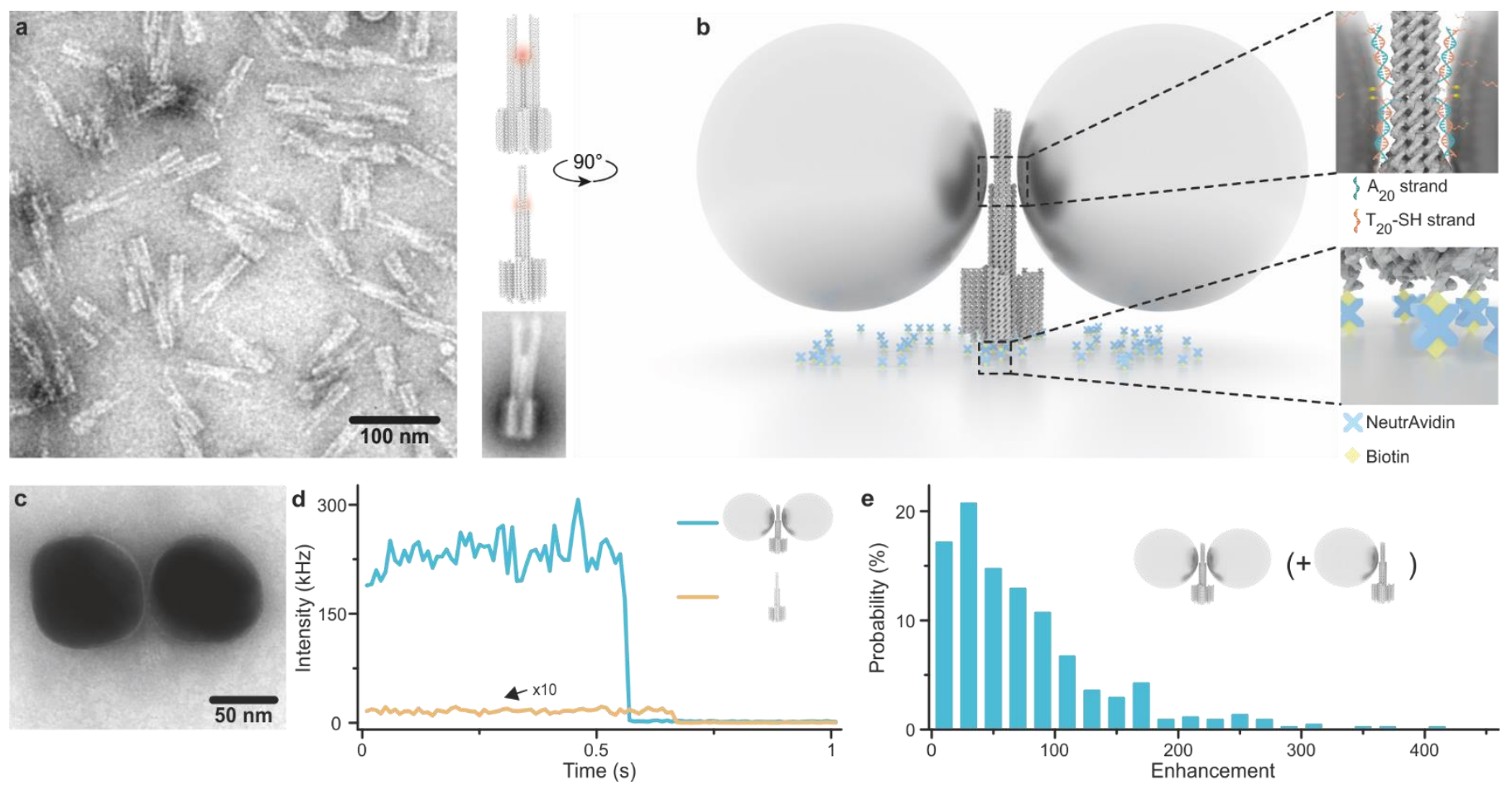

Fig. 1. Concept of the DNA origami nanoantenna with cleared hotspots. a) TEM image (left) and sketch (right) of the DNA origami structure used for the nanoantenna assembly with the position of the plasmonic hotspot indicated in red. A class averaged TEM image of the DNA origami used is shown on the lower right; b) Schematics of NACHOS assembly: the DNA origami construct is bound to the BSA-biotin coated surface via biotin-NeutrAvidin interactions, thiolated DNA-functionalized $100 \mathrm{~nm}$ silver particles are attached to the 
DNA origami nanoantenna via polyadenine $\left(\mathrm{A}_{20}\right)$ binding strands in zipper mode; $\left.{ }^{22} \mathrm{c}\right)$ TEM image of a NACHOS with $100 \mathrm{~nm}$ silver nanoparticles; d) Single-molecule fluorescence intensity transients, measured by confocal microscopy, normalized to the same excitation power of a single Alexa Fluor 647 dye incorporated in a DNA origami (orange) and in a DNA origami nanoantenna with two $100 \mathrm{~nm}$ silver nanoparticles (blue) excited at $639 \mathrm{~nm}$; e) Fluorescence enhancement distribution of Alexa Fluor 647 measured in NACHOS with $100 \mathrm{~nm}$ silver nanoparticles. A total number of 164 and 449 single molecules in the reference (not shown) and NACHOS structures were analysed, respectively.

To utilize the plasmonic hotspot for single-molecule diagnostics we designed a sandwich binding assay capable of detecting a DNA fragment specific to Oxa-48, which is used for the diagnosis of an antibiotic resistant Klebsiella pneumonia infection. ${ }^{23,24}$ Three capture strands specific to the target DNA (Supplementary Table 4) were incorporated, protruding directly into the plasmonic hotspot of the NACHOS. The rationale of using three capturing strands was to optimize the probability of each DNA origami having binding strands accessible to capture the target. ${ }^{25}$ The principle of this assay is illustrated in Fig. 2a: a 17-nt long capture strand is complementary to one half of the 34-nt long target DNA strand. Binding of the target DNA sequence then provides an overhang for the 17-nt long dye-labelled imager strand to be incorporated directly in the plasmonic hotspot where the signal of the reporter dye is amplified by the nanoantenna. In addition, the DNA origami structure is labelled with a single ATTO 542 dye close to the base. Surface scans before incubation with the target and imager strands show green fluorescent spots that represent single NACHOS (Fig. 2b, Supplementary Figure 4). After incubating $\left(2 \mathrm{~h}\right.$ at $\left.37^{\circ} \mathrm{C}\right)$ the NACHOS with the target DNA sequence $(2 \mathrm{nM}$, Supplementary Table 4) as well as with the Alexa Fluor 647-labelled imager strand (6 nM, Supplementary Table 4), the presence of the target DNA could be detected and quantified by counting the number of colocalized green (ATTO 542) and red (Alexa Fluor 647) spots in confocal fluorescence scans (Fig. 2c, Supplementary Figure 4). When the nanoantennas are incubated with the imager strand only (Fig. 2d, 2f, and Supplementary Figure 4), very few co-localized spots are observed. This control demonstrates a very low fraction of false positive signals.
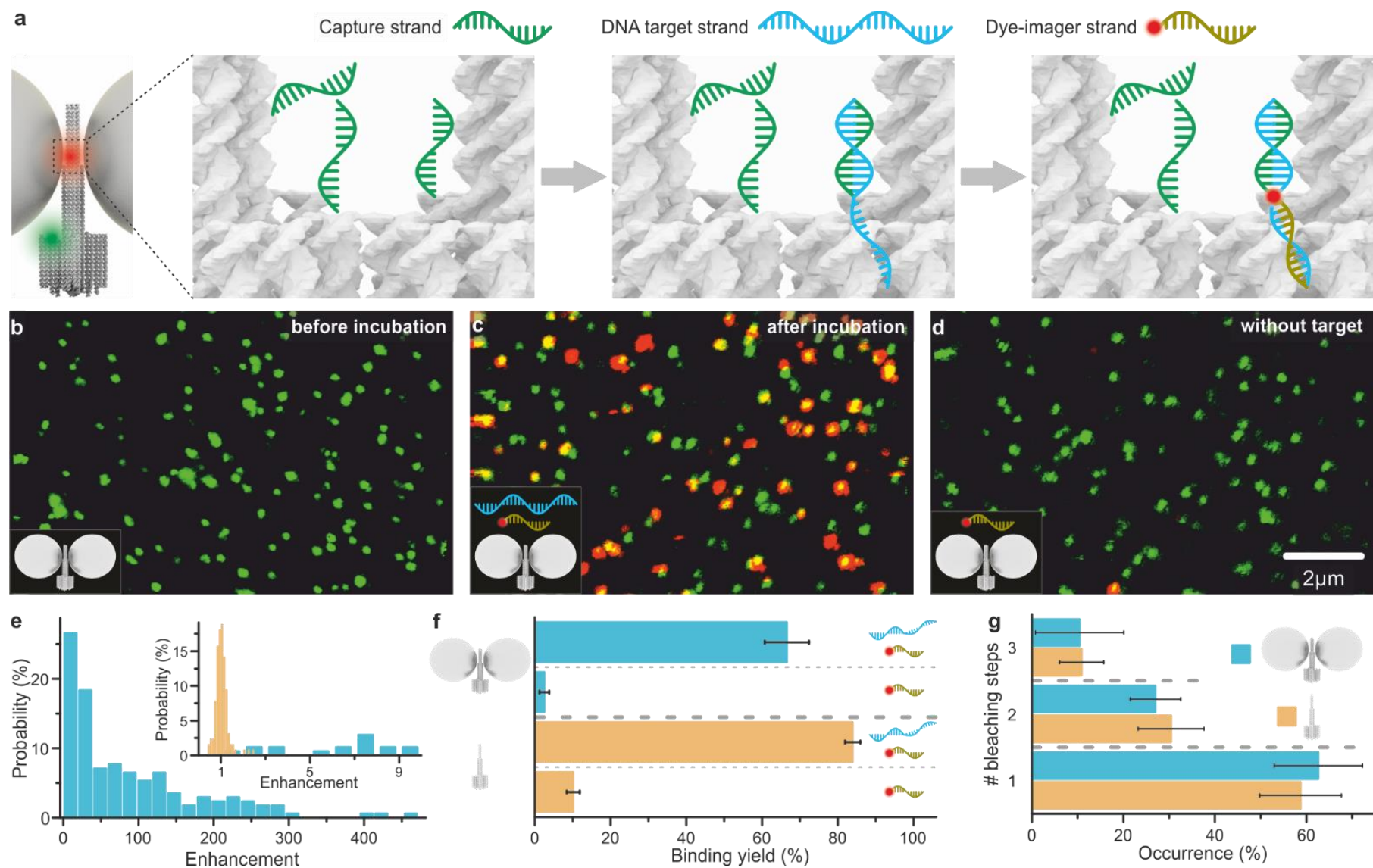

Fig. 2. Single-molecule diagnostic assay with NACHOS. a) Sketch of NACHOS with three capture strands in the hotspot and a green reference dye (ATTO 542) for labelling of the DNA origami. Capture strands are placed in the hotspot region of the DNA origami nanoantenna. Upon incubation, they hybridize with DNA 
target strands specific to Klebsiella pneumonia, exposing a specific, 17-nt long region for the hybridization with the imager strand labelled with Alexa Fluor 647. b,c) Confocal fluorescence images of the NACHOS before and after the incubation with DNA target and imager strands ( $2 \mathrm{nM}$ and $6 \mathrm{nM}$, respectively); d) Confocal fluorescence image of the DNA origami nanoantennas after the incubation with only the DNA imager strand (6 nM); e) Fluorescence enhancement histogram of the sandwich assay in NACHOS. The inset includes a zoom in into the enhancement histogram (region of enhancement values between 0 and 10, blue) overlaid with an enhancement histogram obtained for non-enhanced single Alexa Fluor 647 dyes (orange). A total number of 127 and 169 reference and NACHOS structures were analysed, respectively; f) Binding yield (colocalization yield) obtained for the complete sandwich assay (incubation with $2 \mathrm{nM}$ target and $6 \mathrm{nM}$ imager stands) and for the control experiment (incubation with imager strand only) with (blue) and without (orange) nanoparticles. At least 590 spots were analysed for every sample. Alexa Fluor 647 was excited at $639 \mathrm{~nm}$ and ATTO 542 at $532 \mathrm{~nm}$; g) Distribution of fluorescence bleaching steps observed in fluorescence transients for NACHOS and reference structures. Over 240 structures per sample were analysed. Error bars show the standard deviation from the mean.

Next, we studied the fluorescence enhancement that could be achieved in this single-molecule DNA diagnostics assay (Fig. 2e). Fluorescence enhancement values were calculated by comparing the intensity of Alexa Fluor 647 in NACHOS that contained only one dye incorporated in the hotspot (i.e. displayed singlestep bleaching events in fluorescence transients) to the intensity of single Alexa Fluor 647 dyes incorporated in the reference structure without nanoparticles. As shown in Fig. 2e, fluorescence enhancement values of up to 461-fold could be achieved. One major advantage of using these nanoantennas for the sandwich binding assay is that only the signal originating from the specific binding to the target sequence in the zeptoliter volume of the nanoantenna hotspot is amplified. In contrast, any signal originating from non-specific binding of the imager strand to the DNA origami scaffold or the surface of the glass coverslip is not amplified. The clear differentiation between single-molecule emission amplified by the nanoantenna and the one observed from single fluorescent molecules is illustrated in the inset of Fig. 2e.

We quantified the efficiency of the sandwich binding assay in the reference DNA origami structure without nanoparticles as well as in NACHOS containing $100 \mathrm{~nm}$ silver nanoparticles by calculating the fraction of DNA origami structures containing the target and imager (\% colocalization of green and red spots, Fig. 2f). Binding efficiencies of $66 \%$ and $84 \%$ were measured in NACHOS (blue) and in the reference structures (orange), respectively, confirming that the hotspot accessibility for the target DNA sequence is not significantly compromised. We note that $\sim 10 \%$ higher imager binding yield was observed for the reference structure in the presence as well as in the absence of the target strand, which we attribute to higher non-specific sticking of the imager to the reference structure. We hypothesize this non-specific sticking is related to the single-stranded DNA for nanoparticle binding as unspecific binding is reduced after incorporation of two silver nanoparticles in the full nanoantenna construct (Fig. 2f).

To quantify the number of target molecules incorporated in each nanoantenna hotspot, we performed a single-molecule fluorescence photobleaching analysis (Fig. $2 \mathrm{~g}$ ) which allowed us to determine the number of Alexa Fluor 647 imager strands per DNA origami structure by counting the photobleaching steps in singlemolecule fluorescence transients (Supplementary Figure 5). The majority ( $60 \%$ ) of NACHOS contained one imager strand incorporated in the hotspot, one third of nanoantennas contained two imager strands, while three imager strands were observed in $~ 8-11 \%$ of single-molecule transients. The distribution of bleaching steps obtained for NACHOS as well as for the reference structures (Fig. 2g) further supports the observation that the presence of silver nanoparticles does not obstruct the hotspot accessibility for the DNA target. 
a

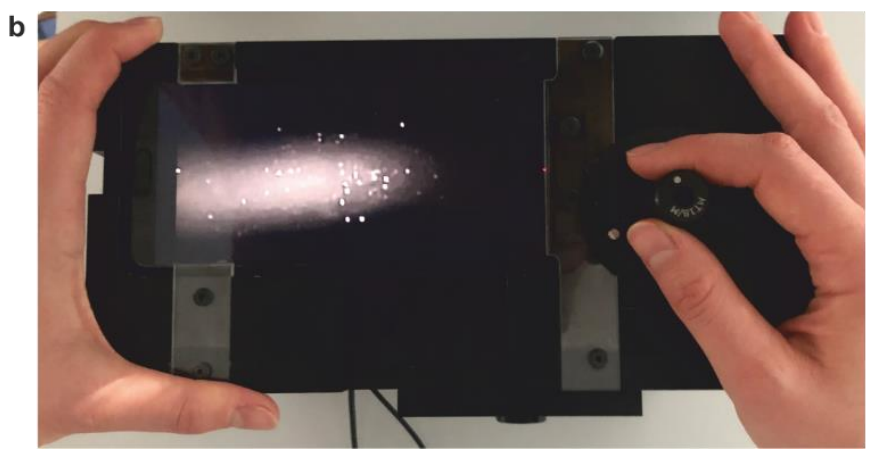

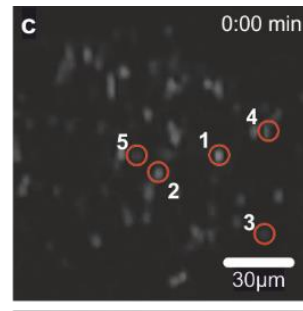
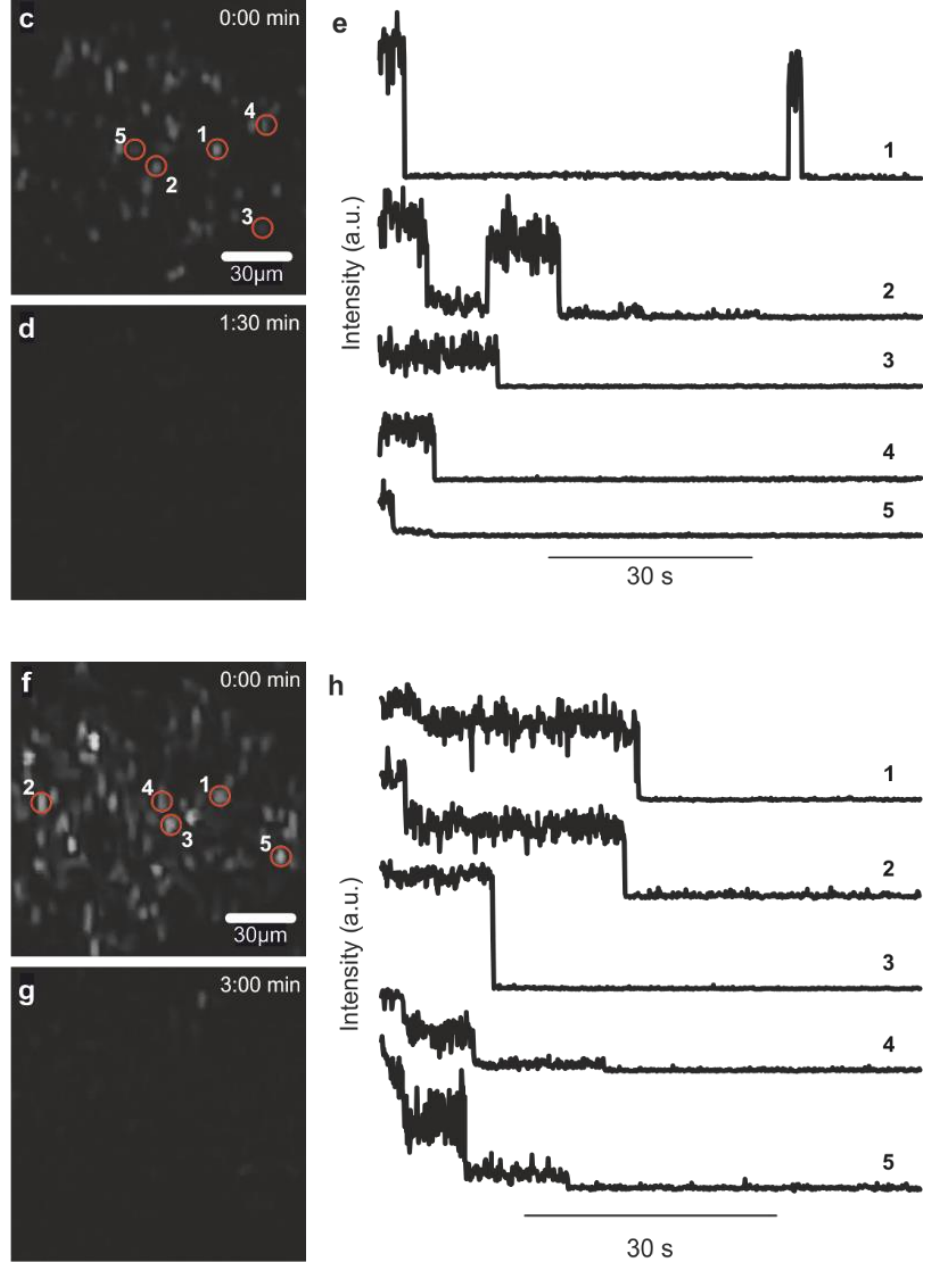

Fig. 3. Single-molecule detection on a portable smartphone microscope. a) Sketch of the portable smartphone microscope with the battery driven $638 \mathrm{~nm}$ laser (red), the focusing lens ( $\mathrm{f}=5 \mathrm{~cm}$ ) (yellow), the microscope coverslip with the sample (blue), the objective lens and the emission filter (brown), and the smartphone camera as detector (green); b) Top view photograph of the portable smartphone microscope; c) Background corrected fluorescence image of NACHOS with $100 \mathrm{~nm}$ silver nanoparticles and a single Alexa Fluor 647 dye. d) Fluorescence image as in c) after illumination of the same area for 1:30 min; e) Exemplary fluorescence transients of a single Alexa Fluor 647 in NACHOS measured on the portable microscope setup. Single bleaching steps of dyes and long-time blinking events are visible. f) Background corrected fluorescence image of NACHOS equipped with a sandwich assay with $100 \mathrm{~nm}$ silver nanoparticles and Alexa Fluor 647 imager strands, g) Fluorescence image as in f) after illumination of the area for 3:00 min; h) Exemplary fluorescence transients of Alexa Fluor 647 in a three-capture-strand DNA origami nanoantenna measured on the portable smartphone microscope. Fluorescence transients with one, two, and three bleaching steps (analogous to single-molecule confocal measurements) were observed. All transients shown in e and $h$ were extracted from a single movie.

Recently, the detection of only 10-16 ATTO 542 molecules was demonstrated using a simple table top setup with a monochrome smartphone camera as detector and a consumer product lens for light collection. ${ }^{26}$ This inspired us that single-molecule detection might be possible on a portable smartphone microscope with non-specialized low-NA optics ${ }^{27-29}$ (see Fig. 3a and 3b). The microscope uses the monochrome camera of a Huawei P20 smartphone for detection, data processing and interfacing and a battery-driven $638 \mathrm{~nm}$ excitation laser with $180 \mathrm{~mW}$ output power. The excitation laser (red in Fig. 3a) is focused on the sample plane at approximately $45^{\circ}$ using a lens with a focal length of $5 \mathrm{~cm}$ to illuminate an elliptical area of $\sim 150 \times 200 \mu \mathrm{m}^{2}$. Fluorescence emission is collected and collimated with a consumer product lens (NA $=0.25,8$ US $\$$, yielding a resolution of $\sim 1.2 \mu \mathrm{m}$ in the red wavelength range), bandpass filtered and focused onto the smartphone detector using the internal lens in the infinite focal distance mode. 
First, we prepared NACHOS with $100 \mathrm{~nm}$ silver nanoparticles and a single Alexa Fluor 647 dye in the hotspot. Taking into account the low resolution of the smartphone microscope, the concentration of NACHOS on the surface was adjusted to a reasonably low density to ensure that only one nanoantenna is present per diffraction limited spot (see Materials and Methods section). To improve the photostability of Alexa Fluor 647 and demonstrate single-molecule bleaching steps, the measurements were carried out in a reducing and oxidizing system (ROXS) ${ }^{30}$ with enzymatic oxygen removal. Upon illumination, multiple bright spots were observed on the smartphone screen (Fig. 3c). In the movies recorded with $80 \mathrm{~ms}$ per frame, single-molecule blinking and bleaching was observed (see Supplementary Videos 1-3) as indicated by the disappearance of spots over time (compare Fig. 3c and d). Extracted fluorescence transients (spots from one movie) are shown in Fig. 3e, demonstrating typical single-molecule behaviour with blinking and single-step bleaching events. These transients represent the first examples of single-molecule fluorescence detection with a portable smartphone microscope and non-dedicated optics bringing single-molecule detection a step closer to point-ofcare settings.

Next, we tested whether the portable smartphone microscope could also be used for the detection of single DNA molecules in analogy to the sandwich assay discussed in Fig. 2. The sandwich assay with three capture strands for the detection of the resistance gene Oxa-48 imaged with the portable smartphone microscope is shown in Fig. 3f. All fluorescence spots acquired on the smartphone camera were photobleached after 3 minutes of video recording (see Supplementary Videos 4-6). The extracted transients (Fig. 3h) exhibit bleaching of the imager strands with 1-3 bleaching steps in accordance with the single-molecule fluorescence transients acquired on the confocal microscope shown in Fig. 2. These results confirm that single-molecule detection of disease-specific DNA can also be performed on our portable smartphone microscope omitting the need for advanced and expensive microscopes. More examples of extracted transients for the sandwich assay with three binding strands as well as for single Alexa Fluor 647 dyes bound to the NACHOS are included in the Supplementary Figures 6 and 7. The photobleaching analysis for the transients from the videos taken on the smartphone microscope is shown in Supplementary Figure 8 and yields similar distributions for single, double and triple photobleaching steps as compared to the data shown in Fig. $2 \mathrm{~g}$, highlighting the ability of the smartphone microscope in combination with NACHOS to provide analytical power comparable to conventional single-molecule microscopy tools.

Self-assembled nanoantennas with a cleared and addressable hotspot represent an inexpensive and versatile platform for fluorescence signal enhancement assays. Single fluorescent molecules immobilized in the hotspot of these newly designed nanoantennas yield higher fluorescence enhancement values than previous approaches with hotspots blocked by the DNA origami nanostructure. NACHOS are robust (see Supplementary Figure 9 for single-molecule data of a similar sample measured over 13 weeks) and importantly, the accessibility of the hotspot for target DNA molecules and imagers is not impaired despite the constricted dimensions. A single-molecule sandwich assay with three capturing strands shows equally high fluorescence enhancement as direct incorporation of a single fluorescent dye in the hotspot and enables singlemolecule detection with amplified signal that facilitates discrimination of single-molecule binding events against an unavoidable background of single-molecule impurities (Fig. 2e inset). The demonstration of singlemolecule assays on a simple battery-operated smartphone microscope makes DNA origami nanoantennas a stepping-stone for democratizing single-molecule detection with cost-effective and mobile devices relevant for point-of-care applications.

1 Michalet, X. et al. Detectors for single-molecule fluorescence imaging and spectroscopy. J. Mod. Opt. 54, 239-239, doi:10.1080/09500340600769067 (2007).

2 Moerner, W. E. \& Fromm, D. P. Methods of single-molecule fluorescence spectroscopy and microscopy. Rev. Sci. Instrum. 74, 3597-3619, doi:10.1063/1.1589587 (2003).

3 Ha, T. \& Tinnefeld, P. Photophysics of Fluorescent Probes for Single-Molecule Biophysics and Super-Resolution Imaging. Annu. Rev. Phys. Chem. 63, 595-617, doi:10.1146/annurev-physchem032210-103340 (2012). 
Ozcan, A. Mobile phones democratize and cultivate next-generation imaging, diagnostics and measurement tools. Lab Chip 14, 3187-3194, doi:10.1039/c4lc00010b (2014). Gill, P. \& Ghaemi, A. Nucleic acid isothermal amplification technologies: a review. Nucleos. Nucleot. Nucl. 27, 224-243, doi:10.1080/15257770701845204 (2008).

Li, J., Macdonald, J. \& von Stetten, F. Review: a comprehensive summary of a decade development of the recombinase polymerase amplification. Analyst 144, 31-67, doi:10.1039/C8AN01621F (2019). Gu, L. et al. Research Progress on Rolling Circle Amplification (RCA)-Based Biomedical Sensing. Pharmaceuticals 11, 35, doi:10.3390/ph11020035 (2018).

Fakruddin, M. et al. Nucleic acid amplification: Alternative methods of polymerase chain reaction. $J$. Pharm. Bioallied. Sci. 5, 245-252, doi:10.4103/0975-7406.120066 (2013).

Kühnemund, M. et al. Targeted DNA sequencing and in situ mutation analysis using mobile phone microscopy. Nat. Commun. 8, 13913, doi:10.1038/ncomms13913 (2017).

10 Taylor, A. B. \& Zijlstra, P. Single-Molecule Plasmon Sensing: Current Status and Future Prospects. ACS Sensors 2, 1103-1122, doi:10.1021/acssensors.7b00382 (2017).

11 Walt, D. R. Optical methods for single molecule detection and analysis. Anal. Chem. 85, 1258-1263, doi:10.1021/ac3027178 (2013).

Li, J.-F., Li, C.-Y. \& Aroca, R. F. Plasmon-enhanced fluorescence spectroscopy. Chem. Soc. Rev. 46, 3962-3979, doi:10.1039/C7CS00169J (2017).

13 Bauch, M., Toma, K., Toma, M., Zhang, Q. \& Dostalek, J. Plasmon-Enhanced Fluorescence Biosensors: a Review. Plasmonics 9, 781-799, doi:10.1007/s11468-013-9660-5 (2014).

14 Punj, D. et al. A plasmonic 'antenna-in-box' platform for enhanced single-molecule analysis at micromolar concentrations. Nat. Nanotech. 8, 512-516, doi:10.1038/nnano.2013.98 (2013). Anger, P., Bharadwaj, P. \& Novotny, L. Enhancement and Quenching of Single-Molecule Fluorescence. Phys. Rev. Lett. 96, 113002, doi:10.1103/PhysRevLett.96.113002 (2006).

16 Curto, A. G. et al. Unidirectional Emission of a Quantum Dot Coupled to a Nanoantenna. Science 329, 930-933, doi:10.1126/science.1191922 (2010).

17 Rothemund, P. W. K. Folding DNA to create nanoscale shapes and patterns. Nature 440, 297-302, doi:10.1038/nature04586 (2006).

18 Acuna, G. P. et al. Fluorescence enhancement at docking sites of DNA-directed self-assembled nanoantennas. Science 338, 506-510, doi:10.1126/science.1228638 (2012).

Ochmann, S. E. et al. Optical Nanoantenna for Single Molecule-Based Detection of Zika Virus Nucleic Acids without Molecular Multiplication. Anal. Chem. 89, 13000-13007, doi:10.1021/acs.analchem.7b04082 (2017).

20 Puchkova, A. et al. DNA Origami Nanoantennas with over 5000-fold Fluorescence Enhancement and Single-Molecule Detection at 25 muM. Nano Lett. 15, 8354-8359, doi:10.1021/acs.nanolett.5b04045 (2015).

21 Vietz, C., Kaminska, I., Sanz Paz, M., Tinnefeld, P. \& Acuna, G. P. Broadband Fluorescence Enhancement with Self-Assembled Silver Nanoparticle Optical Antennas. ACS Nano 11, 4969-4975, doi:10.1021/acsnano.7b01621 (2017).

Vietz, C., Lalkens, B., Acuna, G. P. \& Tinnefeld, P. Functionalizing large nanoparticles for small gaps in dimer nanoantennas. New J. Phys. 18, 045012, doi:10.1088/1367-2630/18/4/045012 (2016).

Hrabak, J., Chudackova, E. \& Papagiannitsis, C. C. Detection of carbapenemases in Enterobacteriaceae: a challenge for diagnostic microbiological laboratories. Clin. Microbiol. Infect. 20, 839-853, doi:10.1111/1469-0691.12678 (2014).

24 Poirel, L., Héritier, C., Tolün, V. \& Nordmann, P. Emergence of oxacillinase-mediated resistance to imipenem in Klebsiella pneumoniae. Antimicrob. Agents Chemother. 48, 15-22, doi:10.1128/aac.48.1.15-22.2004 (2004).

25 Strauss, M. T., Schueder, F., Haas, D., Nickels, P. C. \& Jungmann, R. Quantifying absolute addressability in DNA origami with molecular resolution. Nat. Commun. 9, 1600, doi:10.1038/s41467-018-04031-z (2018).

26 Vietz, C. et al. Benchmarking Smartphone Fluorescence-Based Microscopy with DNA Origami Nanobeads: Reducing the Gap toward Single-Molecule Sensitivity. ACS Omega 4, 637-642, doi:10.1021/acsomega.8b03136 (2019).

27 Wei, Q. et al. Fluorescent imaging of single nanoparticles and viruses on a smart phone. ACS Nano 7, 9147-9155, doi:10.1021/nn4037706 (2013). 
28 Diederich, B., Then, P., Jügler, A., Förster, R. \& Heintzmann, R. cellSTORM-Cost-effective superresolution on a cellphone using dSTORM. PLoS One 14, e0209827-e0209827, doi:10.1371/journal.pone.0209827 (2019).

29 Wei, Q. et al. Imaging and Sizing of Single DNA Molecules on a Mobile Phone. ACS Nano 8, 12725-12733, doi:10.1021/nn505821y (2014).

30 Vogelsang, J. et al. A Reducing and Oxidizing System Minimizes Photobleaching and Blinking of Fluorescent Dyes. Angew. Chem. Int. Ed. 47, 5465-5469, doi:10.1002/anie.200801518 (2008). 


\section{Materials and Methods}

\section{DNA origami}

DNA origami structures were designed in caDNAno $2^{31}$ and assembled and purified using protocols inspired by Wagenbauer et al. ${ }^{32}$ Briefly, $25 \mu \mathrm{L}$ of p8064 scaffold (produced in-house) at $100 \mathrm{nM}$ were mixed with 18 $\mu \mathrm{L}$ of unmodified staples pooled from $100 \mu \mathrm{M}$ original concentration and $2 \mu \mathrm{L}$ of modified staples, pooled from $100 \mu \mathrm{M}$ original concentration. All staples were purchased from Eurofins Genomics GmbH (Germany) - for the exact sequences see Supplementary Table $2.5 \mu \mathrm{L}$ of folding buffer $\left(200 \mathrm{mM} \mathrm{MgCl}_{2}, 50 \mathrm{mM}\right.$ Tris, 50 $\mathrm{mM} \mathrm{NaCl}, 10 \mathrm{mM}$ EDTA) were added and the mixture was subjected to a thermal annealing ramp (see Supplementary Table 1). Samples were purified using $100 \mathrm{kDa}$ MWCO Amicon Ultra filters (Merck KGaA, Germany) with 4 washing steps with a lower ionic strength buffer ( $5 \mathrm{mM} \mathrm{MgCl}, 5 \mathrm{mM}$ Tris, $5 \mathrm{mM} \mathrm{NaCl}, 1$ mM EDTA) for 8 mins at $8 \mathrm{krcf}, 20^{\circ} \mathrm{C}$.

\section{Functionalization of Silver Nanoparticles}

$100 \mathrm{~nm}$ silver nanoparticles (100 nm BioPure Silver Nanospheres (Citrate), nanoComposix, USA) were functionalized with $\mathrm{T}_{20}$ single-stranded DNA oligonucleotides with a thiol modification at the 3'-end (Ella Biotech GmbH, Germany) based on previously described procedures. ${ }^{19} 2 \mathrm{~mL}$ of $0.025 \mathrm{mg} / \mathrm{mL}$ nanoparticle solution in mQ-water was heated to $40{ }^{\circ} \mathrm{C}$ under permanent stirring. $20 \mu \mathrm{L}$ of $10 \%$ Tween ${ }^{\circledR} 20$ and $20 \mu \mathrm{L}$ of a potassium phosphate buffer (4:5 mixture of $1 \mathrm{M}$ monobasic and dibasic potassium phosphate, Sigma Aldrich, USA) were added as well as $10 \mu \mathrm{L}$ of a 2 nmol thiol-modified single-stranded DNA solution (5'-T20-SH-3') and incubated for $1 \mathrm{~h}$ at $40{ }^{\circ} \mathrm{C}$. A salting procedure was then carried out by adding $1 \times \mathrm{PBS}$ buffer containing $3.3 \mathrm{M} \mathrm{NaCl}$ stepwise over $45 \mathrm{~min}$ to the heated and stirred solution, until a final concentration of $750 \mathrm{mM}$ $\mathrm{NaCl}$ was reached. Afterwards, the particles were mixed 1:1 with $1 \times$ PBS $10 \mathrm{mM} \mathrm{NaCl}, 2.11 \mathrm{mM}$ P8709 buffer (Sigma Aldrich, USA), 2.89 mM P8584 buffer (Sigma Aldrich, USA), $0.01 \%$ Tween ${ }^{\circledR} 20$ and $1 \mathrm{mM}$ EDTA. To remove the excess thiolated single-stranded DNA, the solution was centrifuged for 15 min at 2.8 $\mathrm{krcf}$ and $20^{\circ} \mathrm{C}$. A pellet was formed in which the particles were concentrated. The supernatant was discarded, and the washing step was repeated six more times. After functionalization of the silver nanoparticles were diluted in $1 \times \mathrm{TE}$ buffer containing $2 \mathrm{M} \mathrm{NaCl}$ to reach the final extinction of $0.05(0.1 \mathrm{~mm}$ path length) at the extinction maxima on a UV-Vis spectrometer (Nanodrop 2000, Thermo Fisher Scientific, USA).

\section{Solution Synthesis of DNA Origami Nanoantennas for TEM imaging}

To obtain DNA origami nanoantennas in solution, the structures were initially assembled on streptavidincoated magnetic beads (Dynabeads ${ }^{\mathrm{TM}}$ MyOne ${ }^{\mathrm{TM}}$ Streptavidin $\mathrm{C} 1,1 \mu \mathrm{m}$ diameter, $10 \mathrm{mg} / \mathrm{mL}$, Thermo Fischer Scientific, USA). Preparation of magnetic beads: $3.0 \mu \mathrm{L}$ of bead stock solution $\left(\sim 20-30 \times 10^{6}\right.$ beads $)$ were washed three times with $50 \mu \mathrm{L} 1 \times \mathrm{B} \& \mathrm{~W}$ buffer $(0.5 \mathrm{mM}$ EDTA, $5 \mathrm{mM}$ Tris- $\mathrm{HCl}(\mathrm{pH}=8), 1 \mathrm{M} \mathrm{NaCl}, 0.001$ $\% \mathrm{v} / \mathrm{v}$ Tween $\left.{ }^{\circledR} 20\right)$. After removing the supernatant, the beads were diluted in $6.0 \mu \mathrm{L} 1 \times \mathrm{B} \& \mathrm{~W}$ and incubated with $6.0 \mu \mathrm{L}$ of $4 \mu \mathrm{M}$ biotinylated ssDNA (mag1, Supplementary Table 5) for 20 min at room temperature. The functionalized beads were purified from excess of ssDNA by placing the tube on a magnet and discarding the supernatant. The beads were redispersed in $50 \mu \mathrm{L} 1 \times \mathrm{B} \& \mathrm{~W}$ and washed with $1 \times \mathrm{B} \& \mathrm{~W}$ buffer $(3 \times 50 \mu \mathrm{L})$. Immobilization of DNA Origami on Magnetic Beads: DNA origami $(100 \mu \mathrm{L}, 200 \mathrm{pM}$ in $1 \times \mathrm{B} \& \mathrm{~W}$ buffer $)$ with three ssDNA overhang strands on a bottom partially complementary to the sequence on the magnetic beads (mag2, Supplementary Table 5) were incubated together for $2 \mathrm{~h}$ at $37^{\circ} \mathrm{C}$ under gentle shaking $(450 \mathrm{rpm}$, Eppendorf ThermoMixer ${ }^{\circledR} \mathrm{C}$, Eppendorf AG, Germany). Unbound DNA origami was removed by placing the tube on a magnet and discarding the supernatant. The beads were redispersed in $50 \mu \mathrm{L} 1 \times \mathrm{B} \& \mathrm{~W}$ and washed with $1 \times$ B\&W buffer $(5 \times 50 \mu \mathrm{L})$. Binding of $100 \mathrm{~nm}$ silver nanoparticles: Nanoantennas were fabricated on 
magnetic beads by hybridizing with DNA functionalized (5'- $\left.\mathrm{T}_{20}-\mathrm{SH}-3^{\prime}\right) 100 \mathrm{~nm}$ silver nanoparticles to the DNA origamis. For this the supernatant of the with DNA origami coated beads was removed and incubated with $100 \mu \mathrm{L}$ of $100 \mathrm{~nm}$ silver nanoparticles solution using an excess of five nanoparticles per binding site. During the first three hours of incubation, the solution was mixed every $30 \mathrm{~min}$ by gentle pipetting. After overnight incubation at room temperature, the excess of nanoparticles was removed by placing the tube on a magnet and discarding the supernatant. The beads were re-dissolved in $50 \mu \mathrm{L} 1 \times \mathrm{B} \& \mathrm{~W}$ and washed with $1 \times$ B\&W buffer $(5 \times 50 \mu \mathrm{L})$. Cleavage of the assembled structures: Nanoantennas were cleaved from the beads surface by performing a toehold-mediated strand displacement reaction. For cleavage, the supernatant of the bead solution was removed and nanoantennas coated beads were incubated with $20 \mu \mathrm{L} 10 \mu \mathrm{M}$ of the displacement strand (mag3, Supplementary Table 5) for $4 \mathrm{~h}$ at room temperature. Unbound DNA origami nanoantennas were recovered for further use by placing the tube on a magnet.

\section{Transmision electron microscopy (TEM) measurements}

TEM grids (Formvar/carbon, 400 mesh, $\mathrm{Cu}$, TedPella, Inc., USA) were Ar-plasma cleaned and incubated for $60 \mathrm{~s}$ with DNA origami sample $(5 \mu \mathrm{L}, \sim 2-10 \mathrm{nM})$. Grids were washed with $2 \%$ uranyl formate solution $(5$ $\mu \mathrm{L})$ and incubated again afterwards again $4 \mathrm{~s}$ with $2 \%$ uranyl formate solution $(5 \mu \mathrm{L})$ for staining. TEM imaging were performed on a JEM-1100 microscope (JEOL GmbH, Japan) with an acceleration voltage of 80 $\mathrm{kV}$.

\section{Sample preparation on the coverslip for single-molecule confocal measurements}

Microscope coverslips of $24 \mathrm{~mm} \times 60 \mathrm{~mm}$ size and $170 \mu \mathrm{m}$ thickness (Carl Roth $\mathrm{GmbH}$, Germany) were cleaned with UV-Ozone cleaner (PSD-UV4, Novascan Technologies, USA) for $30 \mathrm{~min}$ at $100{ }^{\circ} \mathrm{C}$. Adhesive SecureSeal ${ }^{\mathrm{TM}}$ Hybridization Chambers $(2.6 \mathrm{~mm}$ depth, Grace Bio-Labs, USA) were glued on the clean coverslips. The created wells were washed three times with PBS buffer and then incubated with BSA-biotin $(0.5 \mathrm{mg} / \mathrm{mL}$, Sigma-Aldrich, USA) and NeutrAvidin $(0.2 \mathrm{mg} / \mathrm{mL}$, Thermo Fisher Scientific, USA). The DNA origami (50-100 pM in 1× TE buffer containing $750 \mathrm{mM} \mathrm{NaCl}$ ) was immobilized on the biotin-NeutrAvidn surfaces using covalently attached biotin modifications on the six staple strands on the base. Density of DNA origami nanoantennas on the surface suitable for single-molecule measurements was checked on a microscope. The binding of silver nanoparticles was then performed by incubating the surfaces with $100 \mu \mathrm{L}$ of $\mathrm{T}_{20}$-functionalized silver nanoparticles in $1 \times \mathrm{TE}$ buffer containing $2 \mathrm{M} \mathrm{NaCl}$ overnight at room temperature. To prevent the evaporation of samples, samples were kept in a sealed humidity chambers during the incubation. The nanoantennas were then imaged in $1 \times \mathrm{TE}$ buffer containing $14 \mathrm{mM} \mathrm{MgCl} 2$.

\section{Diagnostic sandwich assay}

To specifically detect the DNA sequence specific to Oxa-48 gene carrying the antibiotic resistance ${ }^{23,24}$, DNA origami nanoantennas were folded containing three specific capture strands (Supplementary Table 4) extruding from the hotspot region of the nanoantenna. After the assembly of the full nanoantenna in the analogous way to the previous section, the samples were incubated with $2 \mathrm{nM}$ target DNA sequence (34 nt) specific to Oxa-48 gene (Supplementary Table 4) as well as $6 \mathrm{nM}$ Alexa Fluor 647 imager strand (17 nt) labelled with Alexa Fluor 647 (Supplementary Table 4) in 1× TE buffer containing $2 \mathrm{M} \mathrm{NaCl}$. The sample was incubated for at $37{ }^{\circ} \mathrm{C}$ for $2 \mathrm{~h}$ and the excess of the target and imager strands was removed by washing six times with $1 \times \mathrm{TE}$ buffer containing $2 \mathrm{M} \mathrm{NaCl}$. The nanoantennas were then imaged in $1 \times \mathrm{TE}$ buffer containing $14 \mathrm{mM} \mathrm{MgCl}$.

\section{Confocal measurements and data analysis}

Confocal fluorescence measurements were performed using a home-built confocal setup based on an inverted microscope (IX-83, Olympus Corporation, Japan) and a $78 \mathrm{MHz}-$ pulsed supercontinuum white light laser (SuperK Extreme, NKT Photonics A/S, Denmark) with selected wavelengths of $532 \mathrm{~nm}$ and $639 \mathrm{~nm}$. The wavelengths are selected via an acousto-optically tunable filter (AOTF, SuperK Dual AOTF, NKT Photonics 
A/S, Denmark). This is controlled by a digital controller (AODS 20160 8R, Crystal Technology, USA) via a computer software (AODS 20160 Control Panel, Crystal Technology, Inc., USA). A second AOTF (AA.AOTF.ns: TN, AA Opto-Electronic, France) was used to alternate $532 \mathrm{~nm}$ and $639 \mathrm{~nm}$ wavelengths if required, as well as to further spectrally clean the laser beam. It is controlled via home-made LabVIEW software (National Instruments, USA). A neutral density filter was used to regulate the laser intensity, followed by a linear polarizer and a $\lambda / 4$ plate to achieve circularly polarized excitation. A dichroic beam splitter (ZT532/640rpc, Chroma Technology, USA) and an immersion oil objective (UPlanSApo 100 $\times$, NA = 1.4, WD $=0.12 \mathrm{~mm}$, Olympus Corporation, Japan) were used to focus the excitation laser onto the sample. Micropositioning was performed using a Piezo-Stage (P-517.3CL, E-501.00, Physik Instrumente GmbH\&Co. $\mathrm{KG}$, Germany). The excitation powers at $639 \mathrm{~nm}$ were set to $200 \mathrm{nW}$ or for $500 \mathrm{nW}$ for the reference samples and to $50 \mathrm{nW}$ for the NACHOS for the recording of the fluorescence transients. These powers were chosen to avoid the saturation in the hotspot. ${ }^{33}$ For the confocal scans, $2 \mu \mathrm{W}$ at $532 \mathrm{~nm}$ and $2 \mu \mathrm{W}$ and $500 \mathrm{nW}$ at 639 $\mathrm{nm}$ were used for the reference and NACHOS samples, respectively. Emitted light was then collected using the same objective and filtered from the excitation light by the dichroic beam splitter. The light was later focused on a $50 \mu \mathrm{m}$ pinhole (Linos AG, Germany) and detected using Single-Photon Avalanche Diodes (SPCM, AQR 14, PerkinElmer, Inc., USA) registered by an TCSPC system (HydraHarp 400, PicoQuant $\mathrm{GmbH}$, Germany) after additional spectral filtering (RazorEdge 647, Semrock Inc., USA for the red channel and BrightLine HC 582/75, Semrock Inc., USA for the green channel). A custom-made LabVIEW software (National Instruments, USA) was used to process the acquired raw data. Background correction was made individually for each transient.

\section{Sample preparation for single-molecule measurements on the smartphone microscope}

The geometry of the smartphone-based microscope required samples to be sealed. To this end, microscope cover slides of $22 \mathrm{~mm} \times 22 \mathrm{~mm}$ size and $170 \mu \mathrm{m}$ thickness (Carl Roth GmbH, Germany) were cleaned with UV-Ozone cleaner (PSD-UV4, Novascan Technologies, USA) for $30 \mathrm{~min}$ at $100{ }^{\circ} \mathrm{C}$. After this a home-made silicon mask with an opening around $15 \mathrm{~mm} \times 15 \mathrm{~mm}$ was glued on a coverslip to create an incubation chamber. Surface functionalization, DNA origami immobilization (5 - $10 \mathrm{pM})$, nanoantenna formation, and the sandwich sensing assay were performed the same was as described above for the NACHOS assembly on coverslips. To seal the samples, the silicon mask was removed, and a double-sided tape was glued on both sides of the cover slide. Then the cover slides were covered with $76 \mathrm{~mm} \times 26 \mathrm{~mm}$ microscope slides $(1 \mathrm{~mm}$ thickness, Carl Roth GmbH, Germany) which were priory cleaned with UV-Ozone cleaner for 30 min at 100 ${ }^{\circ} \mathrm{C}$. Due to limited photostability of Alexa Fluor 647, samples containing the sandwich assay were imaged in the presence of ROXS photostabilization system. A reducing and oxidizing buffer system with enzymatic oxygen removal consisting of $90 \%$ buffer A (14 mM MgCl $2,50 \mathrm{mM}$ Tris, $2 \mathrm{mM}$ Trolox/Troloxquinone and $1 \% \mathrm{w} / \mathrm{v}$ Glucose) and $10 \%$ buffer B (glucose oxidase $(1 \mathrm{mg} / \mathrm{mL}), 0.4 \%(\mathrm{v} / \mathrm{v})$ catalase $(50 \mu \mathrm{g} / \mathrm{mL}), 30 \%$ glycerol, $12.5 \mathrm{mM} \mathrm{KCl}$ ) was used. After this the chambers were sealed with nail polish and imaged after the drying of the glue.

\section{Single-molecule measurements and analysis on the smartphone}

Single-molecule measurements on the smartphone were performed using a home-built portable box. The 638 $\mathrm{nm}$ laser diode (0638L-11A, Integrated Optics, UAB, Lithuania) with an output power $180 \mathrm{~mW}$ that can be driven by various voltage sources (Power plug, mobile power bank, (rechargeable) batteries) was slightly focused ( $\mathrm{f}=50 \mathrm{~mm}$ ) in $45^{\circ}$ angle onto the sample. The fluorescence of the molecules was collected using an objective lens (NA $=0.25$, LS-40166, UCTRONICS, USA) guiding the light to the monochrome camera of the smartphone (P20, Huawei, China) after spectral filtering (BrightLine HC 731/137, Semrock Inc., USA). Videos were recorded via FreeDCam application and analysed with ImageJ (FIJI) using a home written macro to convert MP4 format of the acquired videos to a TIFF format and find the single-molecule signals and extract the fluorescence intensity as a function of illumination time. 


\section{Acknowledgements}

The authors thank Prof. Tim Liedl/Prof. Joachim Rädler for providing access to their facilities especially to the transmission electron microscope. The authors thank Tomas Gisicius for manufacturing the portable smartphone microscope. PT gratefully acknowledges financial support from the DFG (INST 86/1904-1 FUGG, excellence clusters NIM and e-conversion), BMBF (Grants POCEMON, 13N14336, and SIBOF, 03VP03891), and the European Union's Horizon 2020 research and innovation program under grant agreement No. 737089 (Chipscope). GPA gratefully acknowledges support by the Swiss National Science Foundation through the National Center of Competence in Research Bio-Inspired Materials and through grant number 200021_184687. VG and KT acknowledge the support by Humboldt Research Fellowships from the Alexander von Humboldt Foundation. AO acknowledges the support of NSF PATHS-UP and HHMI.

\section{Author contributions}

PT, AO and GPA conceived the project, LG and BL developed the DNA origami structure, KT, VG and MP optimized the solution synthesis procedure, FSe performed the TEM measurements, KT, VG, CC, MP and RY developed the sandwich assay and prepared samples, performed and analysed the measurements on the confocal microscope, CV, LR, MLS, QW, AO, GPA worked on an earlier version of the smartphone microscope, KT, VG, FSt, JZ constructed the portable smartphone microscope, KT, VG, FSt performed and analysed the measurements on the smartphone microscope, KT, VG, LG, FSt, PT wrote the manuscript.

\section{Competing interests}

PT and GPA are inventors on a patent of the described Bottom-up method for fluorescence enhancement in molecular assays, EP1260316.1, 2012, US20130252825 A1.

\section{References}

19 Ochmann, S. E. et al. Optical Nanoantenna for Single Molecule-Based Detection of Zika Virus Nucleic Acids without Molecular Multiplication. Anal. Chem. 89, 13000-13007, doi:10.1021/acs.analchem.7b04082 (2017).

23 Hrabak, J., Chudackova, E. \& Papagiannitsis, C. C. Detection of carbapenemases in Enterobacteriaceae: a challenge for diagnostic microbiological laboratories. Clin. Microbiol. Infect. 20, 839-853, doi:10.1111/1469-0691.12678 (2014).

24 Poirel, L., Héritier, C., Tolün, V. \& Nordmann, P. Emergence of oxacillinase-mediated resistance to imipenem in Klebsiella pneumoniae. Antimicrob. Agents Chemother. 48, 15-22, doi:10.1128/aac.48.1.15-22.2004 (2004).

31 Douglas, S. M. et al. Rapid prototyping of 3D DNA-origami shapes with caDNAno. Nucleic Acids Res. 37, 5001-5006, doi:10.1093/nar/gkp436 (2009).

32 Wagenbauer, K. F. et al. How We Make DNA Origami. ChemBioChem 18, 1873-1885, doi:10.1002/cbic.201700377 (2017).

33 Grabenhorst, L., Trofymchuk, K., Steiner, F., Glembockyte, V. \& Tinnefeld, P. Fluorophore photostability and saturation in the hotspot of DNA origami nanoantennas. Methods Appl. Fluoresc. 8, 024003, doi:10.1088/2050-6120/ab6ac8 (2020). 
bioRxiv preprint doi: https://doi.org/10.1101/2020.04.09.032037. The copyright holder for this preprint (which was not peer-reviewed) is the author/funder. All rights reserved. No reuse allowed without permission. 